\section{EDITOR:}

- C.J. Eales, PhD

University of the Witwatersrand

\section{Assistant Editor:}

- A. Stewart, MSc

University of the Witwatersrand

\section{EdTTORIAL BOARD:}

NATIONAL MEMBERS

- S.L. Amosun, PhD

University of the Western Cape

- P. Gounden, PhD

University of Durban-Westville

- M. Papadoupolos, MSc

University of Pretoria

- M. Faure, MPhil

University of Stellenbosch

- N. Mbambo, MSc

MEDUNSA

\section{INTERNATIONAL MEMBERS}

- A. Akinpelu, $\mathrm{PhD}$

University College Hospital,

Ibadan, Nigeria

- T.H.A. Kolobe, PhD

University of Illinois, Chicago

- K. Shepard, PhD

Temple University, Philadelphia

- C. Partridge, $\mathrm{PhD}$

University of Kent, Canterbury

\section{Review Panel:}

- Amosun, Dele

- Bester, Ria

- Bischof, Faith

- Bowerbank, Pat

- Crous, Lynette

- David, Helen

- Diener, Ina

- Eksteen, Carina

- Eisenberg, Mashe

- Faure, Mary

- Fearnhead, Lynn

- Frieg, Annette

- Giraud, Jill

- Hunter, Linda

- Irwin-Carruthers, Sheena

- Jelsma, Jennifer

- Kemp, Stephanie

- M'kumbuzi, Vyvienne

- Mothabeng, Joyce

- Mpofu, Ratie

- Papadopoulos, Magda

- Paulsen, Tom

- Potterton, Joanne

- Puckree, Lina

- Smith, Elsa

- Van Rooijen, Tanya

\title{
SALUTING Diversity
}

$\mathrm{T}_{\mathrm{b}}^{\mathrm{h}}$ he healthcare professions have been called upon by government to address diversity in the student population as well as clinical and teaching personel. Diversity of gender, culture and religion are accepted and valued due to the enrichment it brings to our profession and lives at all levels. Diversity pertaining to ideas and insight are of equal value.

According to Longman's dictionary diversity is synonomous with variety and different, both words that has a promise of excitement, stimulus and potential of something more than the ordinary. It has the potential of growth and of deeper understanding. Within a culture of diversity there has to be acceptance and appreciation of differences and similarities which enhances the process of working together towards a common goal. This common goal in the caring professions is ultimately the wellbeing of the patient as well as the wellbeing of the profession itself.

Diversity is found within the profession of physiotherapy at various levels, for example between the public and private sectors, academic and clinical settings and in the various fields of interest. Within these settings there are examples where physiotherapists have found sufficient grounds for working together by forming partnerships, as in the more recent merger between Physio Forum and the SASP with the obvious positive results.

There is however much more scope for working together as partners towards common goals within the profession. It is evident in many spheres of physiotherapy that there is not sufficient professional trust and acknowledgement of each other's strengths and contributions. Derogatory remarks are heard between different interest groups and individual physiotherapists and it becomes evident in discussions that there is often a lack of appreciation of the contribution individuals and different interest groups make towards the growth and standing of the profession.

Being a relatively small and beleagered profession, we can hardly afford discord and division. Our strength lies in unity, sharing and acceptance of the unique contribution each and everyone can make. It lies in utilizing potential within groups and the profession as a whole and supporting individuals to reach their potential. It lies in giving recognition to each other and saluting diversity.

RIA BESTER 